\title{
Design and Implementation of Voltage Peak Detection Based on Fourier Analysis
}

\author{
Hangyu Guo, Shiyao Cui ${ }^{*}$ and Xingxing Xu \\ School of Information Science and Engineering, University of Jinan, Shandong 250022, China. \\ *Corresponding author
}

\begin{abstract}
In order to measure the peak value of the sinusoidal wave more conveniently, we did a full-wave rectification to the sinusoidal wave, then analyzed its frequency spectrum by using Fourier analysis. By researching the relationship between the $\mathrm{DC}$ component in the spectrum and the peak voltage, this paper provided a voltage peak value detect circuit based on the Fourier analysis. The detect circuit is including precision full wave rectifier circuit, second-order RC filter circuit and an ADC converter. Also this paper gives a technique based on the regression analysis in order to reduce the error due to the nonlinear characteristics and the manufacture errors between the real components. By doing the regression analysis of the raw data, which using MATLAB, the circuit will allow measuring error within $2 \%$.
\end{abstract}

Keywords-fourier analysis, DC component, precision rectifier, regression analysis

\section{INTRODUCTION}

Peak detection technology is widely used in the field of signal processing and electrical engineering. Nowadays, most of the technologies are based on the peak holding technology, which usually has a complex circuit. In order to reduce this complexity circuit, this paper put forwards a new circuit design which bases on the amplifier and the Fourier analysis. By processing the sinusoidal signals with the process of full-wave rectification and analyzing it with Fourier analysis, we can get the relationship between the DC component in the frequency spectrum and the peak voltage. This relationship provides a new idea for peak detection.

\section{THEORY OF THE PEAK DETECTION}

The implementation of the peak detection in this paper is based on the Fourier analysis. By Fourier series expansion, any signal can be expanded into the sum of the DC component and many sinusoidal and cosine terms. By researching the relationship between the DC component in the spectrum and the peak voltage, we can detect the peak voltage of the input sinusoidal signal.

But after doing Fourier analysis to the sinusoidal signal, we found that there's no DC component in single-frequency sinusoidal signal, or there is no necessary relationship between DC component and peak value. So it is necessary to transform the sinusoidal signal at first. We assume that

$$
U=U_{M} \cos \omega_{0} t
$$

is the sinusoidal signal that needs to be measured. $U_{\mathrm{M}}$ is the peak voltage, and the $\omega_{0}$ is the angular frequency. Then make the signal "(1)" pass through the full wave rectifier circuit, the signal will change to

$$
U^{\prime}=U_{M}\left|\cos \omega_{0} t\right|
$$

By doing the Fourier expansion of the "(2)", the result is

$$
U^{\prime}=A_{0}+\sum_{n=1}^{\infty} A_{n} \cos n \omega_{0} t
$$

In the "(3)", the $A_{0}$ is DC component. Its value is calculated as

$$
A_{0}=\frac{4 \omega_{0}}{\pi} \int_{-\frac{\pi}{2 \omega_{0}}}^{\frac{\pi}{2 \omega_{0}}} U_{M}\left|\cos \omega_{0} t\right| \mathrm{d} t=\frac{2 U_{M}}{\pi}
$$

Through"(4)", it is clear that there is a linear relationship between the DC component and the peak voltage. The proportional coefficient is $2 / \pi$.

In order to obtain this DC component, a low-pass filter can be designed. The upper limit cut-out frequency $f_{\mathrm{TH}}$ of it needs to satisfy the inequality "(5)".

$$
\frac{\omega_{0}}{2 \pi}<f_{T H}<\frac{\omega_{0}}{\pi}
$$

In the practical using, considering that the limitation of the $\mathrm{AD}$ converter input voltage, it is necessary to attenuate or amplify DC component voltage $A_{0}$. Set the voltage conversion coefficient to $k$, and $\mathrm{ADC}$ input voltage is $U_{\mathrm{AD}}=k A_{0}$. Taking consider in "(4)", we can get the relationship between $U_{\mathrm{AD}}$ and $U_{\mathrm{M}}$ is 


$$
U_{M}=\frac{\pi}{2 k} U_{A D}
$$

So, all of the processing can be divided into 5 steps: signal input, full wave rectification, low-pass filter, voltage transform and peak voltage output (Figure I). In these steps, full wave rectification and low-pass filter is based the Fourier analysis theory, voltage transform is based the relationship between DC component and peak voltage.

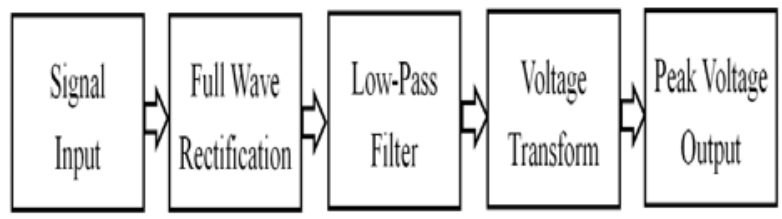

FIGURE I. FUNCTIONAL BLOCK DIAGRAM OF THE PEAK DETECTION

\section{CIRCUIT DESIGN}

\section{A. Design of Precision Rectifier Circuit}

Full wave rectifier needs to use rectifier circuit to convert input sinusoidal signal into pulsating DC signal. But the normal bridge full-wave rectifier circuit will cause distortion because of the existence of the diode opening voltage especially at the circumstance of the small signal input, which leads errors. To minimize this impact as possible, A precision rectifier circuit [2](Figure II) is needed. Also we added input buffer to the basic circuit to improve input resistance.

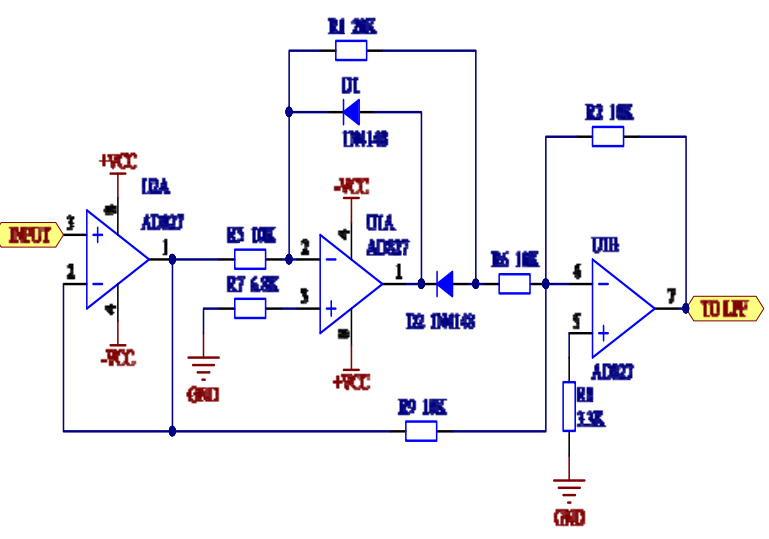

FIGURE II. PRECISION RECTIFIER CIRCUIT

It was found that during experiment that through the impact of diode opening voltage is reduced, the influence of gain bandwidth of operational amplifier also affects the distortion of circuit. If choose an amplifier with small gain bandwidth $(<1.1 \mathrm{MHz})$, the output signal can be seriously distorted by the amplifier that adds measuring error. But if choose a high speed amplifier (Gain bandwidth $>27 \mathrm{MHz}$ ), the operating frequency of the circuit can reach up to more than $500 \mathrm{KHz}$, which greatly expanding its application range.

\section{B. Design of Low-Pass Filtering Circuit}

Low-pass filtering is used to obtain the DC component $A_{0}$ in the "(3)" which relate to the peak voltage. After comprehensive consideration, the second-order passive RC low-pass filter network (Figure III) is selected to form a lowpass filter.

According to reference [1], by solving the system function and frequency response of the second-order filter with Laplace transform method, the high cut-off frequency $f_{\mathrm{TH}}$ of the secondorder filter is

$$
f_{T H}=\frac{0.37}{2 \pi R C}
$$

In the practical circuit, we need to choose the value of resistor-capacitor not only by the calculation but also the value of actual components.

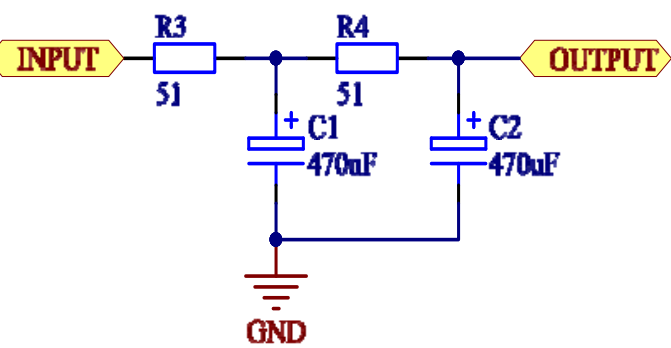

FIGURE III. SECOND-ORDER PASSIVE RC LOW-PASS FILTER

\section{Design of the Voltage Transform Function}

Voltage conversion function is based on the AD converter. Also, by selecting the ADC with high resolution will reduce the measuring error to a certain extent. The ADC output voltage $U_{\mathrm{AD}}$ can be described by the "(8)".

$$
U_{A D}=\frac{N}{2^{B}} V_{r e f}
$$

In this function, $V_{\text {ref }}$ means reference voltage, $N$ means the raw converting data collected by the ADC, $B$ means the ADC resolution. The basic relationship between the peak voltage and the ADC output voltage satisfy "(5)". In order to keep each sampling voltage behaves universally, we sampled each voltage many times and get many peak voltage $U_{\mathrm{Mi}}$. After that, we averaged them and recorded the average peak voltage $\bar{U}_{M}$ as the result during each measuring times. The $\bar{U}_{M}$ can be calculated by the "(9)".

$$
\bar{U}_{M}=\frac{1}{n} \sum_{i=1}^{n} U_{M i}=\frac{1}{n} \sum_{i=1}^{n} \frac{\pi V_{r e f}}{2^{B+1} k} N_{i}
$$




\section{Measure to Cut Down the Errors}

In order to reduce the influence of the errors due to the nonlinear characteristics and the manufacture errors between the real components, we used the MATLAB software to carry out the regression analysis of the measurement results by the non-linear least squares method. Based on this, we update the relationship between the peak voltage UM and the output voltage UAD. Figure IV is the fitting image based on the quadratic polynomial regression. After the regression analysis, the measuring error is significantly lower than before (Table II).

\section{MEASURING RESUltS AND ERROR ANALYSIS}

After the text edit has been completed, the paper is ready for the template. Duplicate the template file by using the Save As command, and use the naming convention prescribed by your conference for the name of your paper. In this newly created file, highlight all of the contents and import your prepared text file. You are now ready to style your paper; use the scroll down window on the left of the MS Word Formatting toolbar.

\section{A. Measuring Results}

By using the method presented in this paper, the measurement results at $100 \mathrm{KHz}$ are recorded in the table as follows (Table I ).

TABLE I. MEASURING RESULTS AND ERRORS

\begin{tabular}{|c|c|c|}
\hline al Voltage & Measured Voltage & Errors \\
\hline $100 \mathrm{mV}$ & $98.4 \mathrm{mV}$ & $-1.59 \%$ \\
\hline $200 \mathrm{mV}$ & $196.4 \mathrm{mV}$ & $-1.86 \%$ \\
\hline $300 \mathrm{mV}$ & $294.8 \mathrm{mV}$ & $-1.77 \%$ \\
\hline $400 \mathrm{mV}$ & $395.1 \mathrm{mV}$ & $-1.25 \%$ \\
\hline $500 \mathrm{mV}$ & $499.5 \mathrm{mV}$ & $-0.10 \%$ \\
\hline $600 \mathrm{mV}$ & $606.9 \mathrm{mV}$ & $1.13 \%$ \\
\hline $700 \mathrm{mV}$ & $714.7 \mathrm{mV}$ & $2.06 \%$ \\
\hline $800 \mathrm{mV}$ & $820.7 \mathrm{mV}$ & $2.53 \%$ \\
\hline $900 \mathrm{mV}$ & $916.3 \mathrm{mV}$ & $1.78 \%$ \\
\hline
\end{tabular}

B. Analysis of the System Errors

In the process of the test, we found that there is some proportional error behaved in the measurement results. Also, to some extent, it shows non-linear feature. We qualitatively analyzed the error in two aspects.

Firstly, the non-linearity of the real operational amplifiers, which lead to the incomplete linear relationship between the output voltage $U_{\mathrm{AD}}$ and the input peak voltage $U_{\mathrm{M}}$, resulted in the non-linearity error in the measurement results.

Secondly, the unsatisfactory parameters of actual passive devices resulted in the unsatisfactory circuit parameters, which made the results behave stable offset between the measured value and the real value without considering the non-linear error.

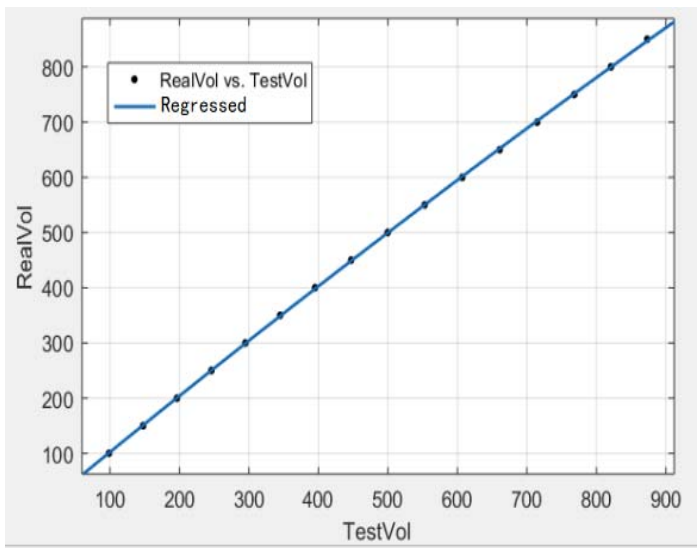

FIGURE IV. FITTING IMAGE

TABLE II. MEASURING RESULTS AFTER REGRESSING

\begin{tabular}{|c|c|c|}
\hline Real Voltage & Fitting Voltage & Errors \\
\hline $100 \mathrm{mV}$ & $100.7 \mathrm{mV}$ & $0.72 \%$ \\
\hline $200 \mathrm{mV}$ & $200.5 \mathrm{mV}$ & $0.24 \%$ \\
\hline $300 \mathrm{mV}$ & $299.2 \mathrm{mV}$ & $-0.26 \%$ \\
\hline $400 \mathrm{mV}$ & $398.1 \mathrm{mV}$ & $-0.47 \%$ \\
\hline $500 \mathrm{mV}$ & $499.5 \mathrm{mV}$ & $-0.11 \%$ \\
\hline $600 \mathrm{mV}$ & $601.7 \mathrm{mV}$ & $0.29 \%$ \\
\hline $700 \mathrm{mV}$ & $702.6 \mathrm{mV}$ & $0.37 \%$ \\
\hline $800 \mathrm{mV}$ & $799.9 \mathrm{mV}$ & $-0.02 \%$ \\
\hline $900 \mathrm{mV}$ & $886.0 \mathrm{mV}$ & $-1.58 \%$ \\
\hline
\end{tabular}

V. SUMMARY

To sum up, the method is practicable by doing Fourier series analysis of the signal which generate from the full-wave rectification by the sinusoidal wave, so as to establish the relationship between the peak voltage of the input sinusoidal wave and the DC component to implement peak value measurement. Experiments showed that this design can keep good performance from $20 \mathrm{~Hz}$ to $500 \mathrm{KHz}$ on the basis of properly operation amplifier select. However, this design could not work at low frequency circumstances, at which the phenomenon of the charging and discharging effect of capacitance behaves so obvious that low-order harmonics are included after the low-pass filter, which produces measuring error obviously. 
By this method, the peak voltage of other signal such as triangular wave and rectangular wave can also be detected.

\section{REFERENCES}

[1] Shibai Tong, Yingcheng Hua.Fundamentals of Analog Electronics Technology, fifth ed., Higher Education Press., Beijing, 2015, pp. 308437.J. Clerk Maxwell, A Treatise on Electricity and Magnetism, 3rd ed., vol. 2. Oxford: Clarendon, 1892, pp.68-73.

[2] Yingbo Lee, Zengxian Xin, An Hu, Jiesu Liu, Simulation Design and Exploration of High Speed Precision Rectifier Circuit, J. Guidance \& Fuze. 36 (2015) 46-49 